\title{
Classification of Latent Fingerprints Using Neural Networks
}

\author{
Hamid JAN, Amjad ALI \\ Sarhad University of Science and Information Technology, \\ LandiAkhun Ahmad, Hayatabad Link, Ring Road, Peshawar, Pakistan \\ hod.csit@suit.edu.pk, amjadali@suit.edu.pk
}

\begin{abstract}
To classify latent fingerprint images by using papillary patterns, the proposed method applies the Gabor filter, Haar and Daubechies wavelet transformations, and a multilevel neural network. Numerical experiments were performed, and the results of the proposed solution were compared. The results have shown that we can effectively classify latent fingerprints by applying in combination the Gabor filter, Daubechies wavelet transform of the fifth level, and a neural network.
\end{abstract}

Key words:Haar and Daubechies wavelet transformations, latent fingerprints, Gabor filter, papillary pattern

\section{Introduction}

Fingerprints are an important feature of human kind. Fingerprint features do not change after a person reaches 14 years of age (Jain et al., 2014; Jain et al., 1999; Maltoni et al., 2009). Owing to the invariance and uniqueness of fingerprints; they are used as a government resource for crime investigation. However, the fingerprints collected from crime scenes, latent fingerprints are often smudgy and have a low resolution. Moreover, they typically cover a small portion of the finger; not the whole finger. Fig. 1 shows the fingerprints obtained by three different methods.

For more than a hundred years, fingerprints have been the most widely used tool for human identification. Thus, owing to the existence of vast fingerprint databases, fingerprint classification has become a critical issue for the automatic fingerprint identification system (AFIS) and automatic fingerprint recognition system (AFRS) (Jain et al., 2014). Classification of fingerprints reduces the computation complexity by minimizing the quantity of candidate matches under study; hence, an effective indexing method can notably enhance the process. There are two types of fingerprint features: global and local. 


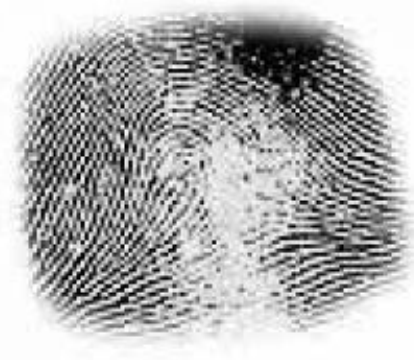

(a)

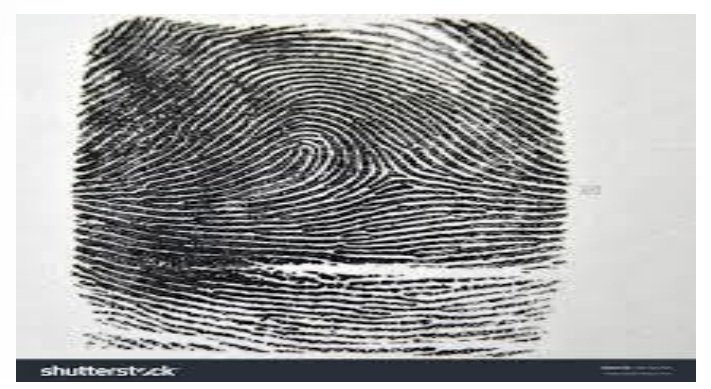

(b)

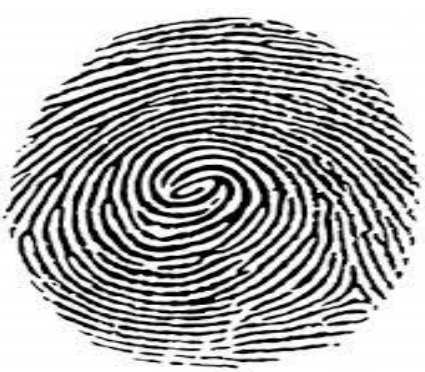

(c)

Fig. 1. Fingerprints obtained by different acquisition methods.

(a) Latent(National Institute of Standards and Technology, 2017),

(b) Rolled ink(National Institute of Standards and Technology, 2017),

(c) live-scan(Fingerprint Verification Competition, 2002).

\subsection{Global Features}

The image area is a fragment of the fingerprint in which all global signs are located(Jain et al., 2014; Jain et al., 1999; Maltoni et al., 2009).Fingerprints can be read and classified based on the image area information. It is thus more effective to identify people using both features. Global features are composed of ridges and valleys. The ridges are the lines; the valleys are the spaces between lines. The important points are the core and delta. The core is the fingerprint centre;the delta is the centre point where three different direction flows meet. The ridges and valleys provide important information for the classification and matching of fingerprints.

Papillary patterns are divided into three types: arches, loops and curls (Wilson et al., 1993). Arches are relatively infrequent and occupy from $5 \%$ to $10 \%$ of all fingerprints. Loops are found in most people and comprise $60 \%$ to $65 \%$ of all fingerprints. Curls are found in $30 \%$ of all fingerprints. Fig. 2 shows the different types of papillary patterns. 


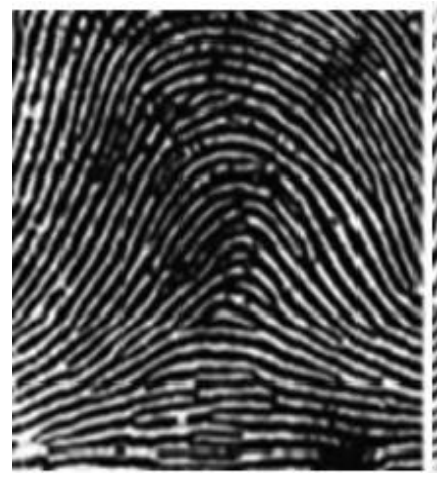

(a)

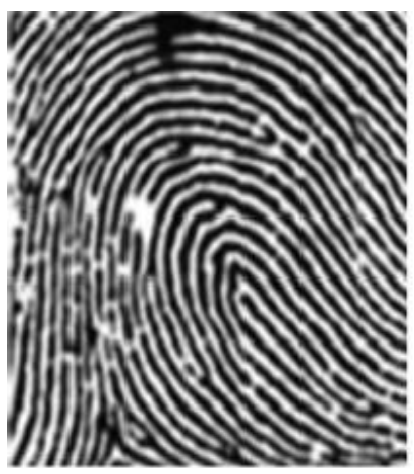

(d)

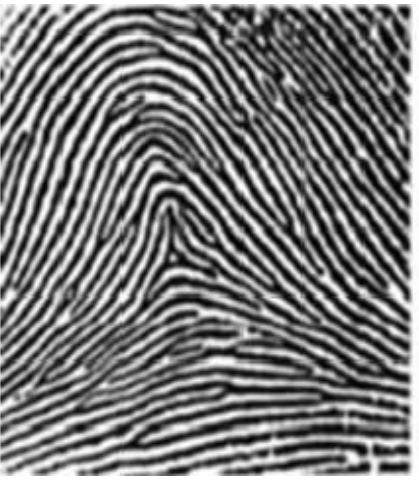

(b)

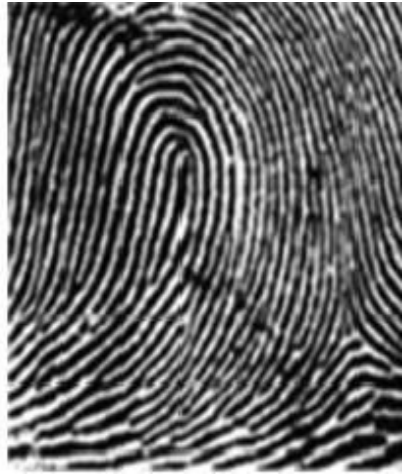

(c)

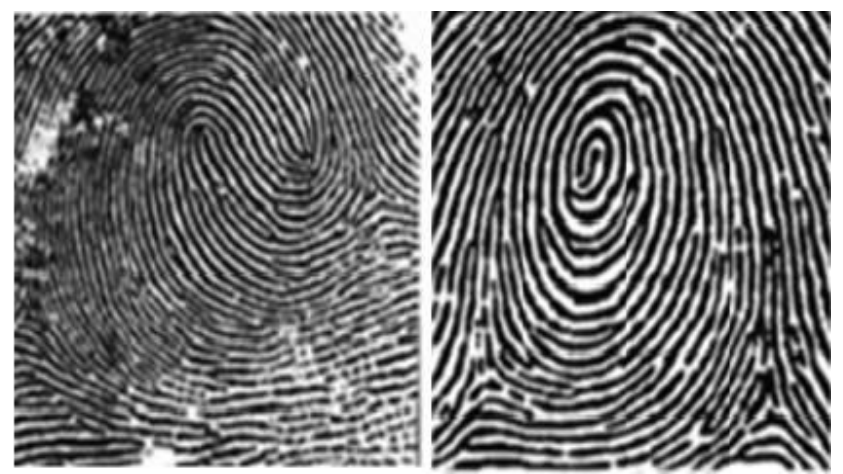

(e)

(f)

Fig. 2. Types of papillary patterns:

a) simple arch, b) three-armed arch, c)left loop,

d) right loop, e) double loop, f) curl.

\subsection{Local Features}

Fingerprint lines are not straight lines (Wilson et al., 1993); they are often broken, branched, change directions, and have tears. The points in which the lines end, fork, or change direction are called minutiae points. These minutiae points provide unique fingerprint information for person identification. The minutiae points are shown in Fig.3. 

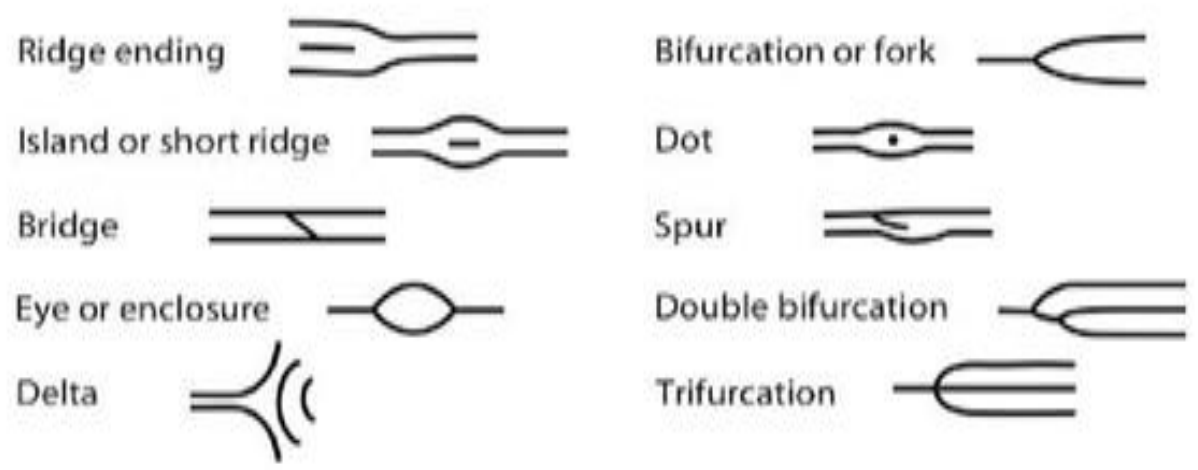

Fig. 3. Minutiae points.

\section{Related Work}

To date, extensive research has been conducted on fingerprint imaging. In this section, we identify some studies conducted on fingerprint classification accordingly, fingerprints are divided into several predefined classes. Classification of latent fingerprints requires considerable time and effort. Attempts have been made to systematize the classification procedure using conventional imaging techniques; nonetheless, very few have been created through law enforcement agencies because of their limited achievements in solving the problem. The increasing interest in neural networks in recent years has wedged the courtesy of those who were confused in the recognition of fingerprints when they begin to distinguish between the potential rewards of the neural network method.

AlShemmary (Najim and AlShemmary, 2012) defined a method for the classification of fingerprints based on both individual characteristics and the study of a neural network. Noise exists in many fingerprint images used by researchers, including those from the NIST databases. Thus, it is difficult to obtain the correct number and position of features, such as core or delta points, which are widely used in existing methods of structural classification. Moreover, several transitional solutions for fingerprint organization that employ neural network as a decision-making stage were presented by Patil and Suralkar (2012). In their approaches, the neural network performs the matching procedure and is effectively industrialized to recognize and classify the fingerprint algorithm by inverse processing. Their solutions improved the accuracy of fingerprint quality classification more efficiently than others.

In addition, an algorithm of perception and correction of skin distortions, based on a single fingerprint image, was presented by $\mathrm{Si}$ et al. (2015). Distortion recognition was considered a classification problem in two classes since the enumerated map of the ridge orientation and the fingerprint epoch map were used as a vector function. Furthermore, 
the SVM classifier performed the organization. Straightening of distortion was observed as a regression problem when the input signal was a misleading fingerprint and the output was a distortion field. Moreover, Al-Nima et al. (2016) proposed a method of human authentication in which finger texture (FT) patterns were used.

To distinguish fingers from manual images, a reliable and automatic method of finger extraction was used. To extract new functions, the extended binary local line template (ELLBP) was employed. The proposed information was built into the poorly displayed areas of the FT, thereby completing the missing elements. The classification was carried out by creating a probabilistic neural network (PNN).Al-Nima et al. (2016) also defined an approach of authentication based on finger texture (FT). FT functions of images including four fingers (index, middle, ring, and pinky) are extracted from the contactless image with a low resolution. To improve FT efficiency, a new enhancement to image functions (IFE) was used to improve FT. The resulting image of the function is segmented, and a probabilistic neural network (PNN) is reasonably recognized for classification.

\section{Fingerprint Classification Methodology}

In this paper, a method is proposed for classifying fingerprint images according to the types of papillary patterns by applying the Gabor filter, wavelet transformation, and multilevel neural network. The functional scheme of the proposed method is shown in Fig.4.

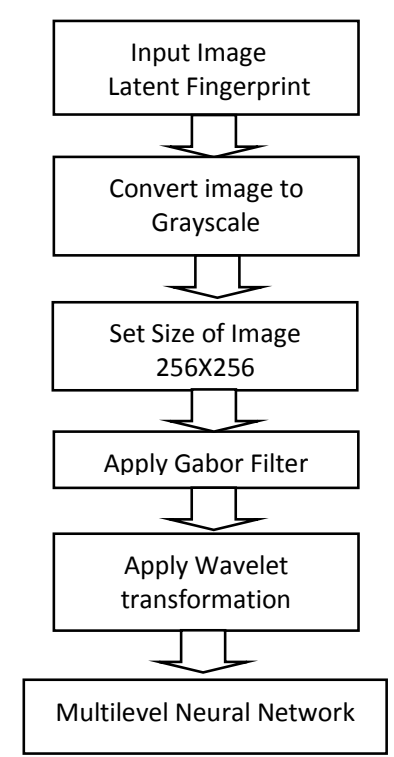

Fig. 4. Functional diagram of the proposed method. 


\subsection{Gabor Filter}

The impulse response of the linear Gabor filter is represented as a product of the Gaussian function for a harmonic function:

$$
\begin{gathered}
g(x, y)=\operatorname{Gauss}\left(x^{\prime}, y^{\prime}\right) \cos \left(\frac{2 \pi x^{\prime}}{\lambda}+\varphi\right), \\
\operatorname{Gauss}\left(x^{\prime}, y^{\prime}\right)=e^{-\left(\frac{x^{\prime 2}}{2 \pi \sigma^{2} x}+\frac{y^{2} y^{\prime}}{2 \pi \sigma^{2} y}\right),} \\
x^{\prime}=x \cos \theta+y \sin \theta \\
y^{\prime}=-x \sin \theta+y \cos \theta
\end{gathered}
$$

where $\lambda$ is the wavelength, and $\varphi$ is the phase. In addition, angle $\theta$ indicates the orientation of the normal to the parallel bands of the Gabor function, and $\gamma$ is the compression coefficient. Changing orientation $\theta$ makes it possible to change the edge detection direction. Examples of the Gabor filter with orientations in the range of 0 to $\pi$ in steps of $\pi / 16$ are shown in Figs. 5 to 7.
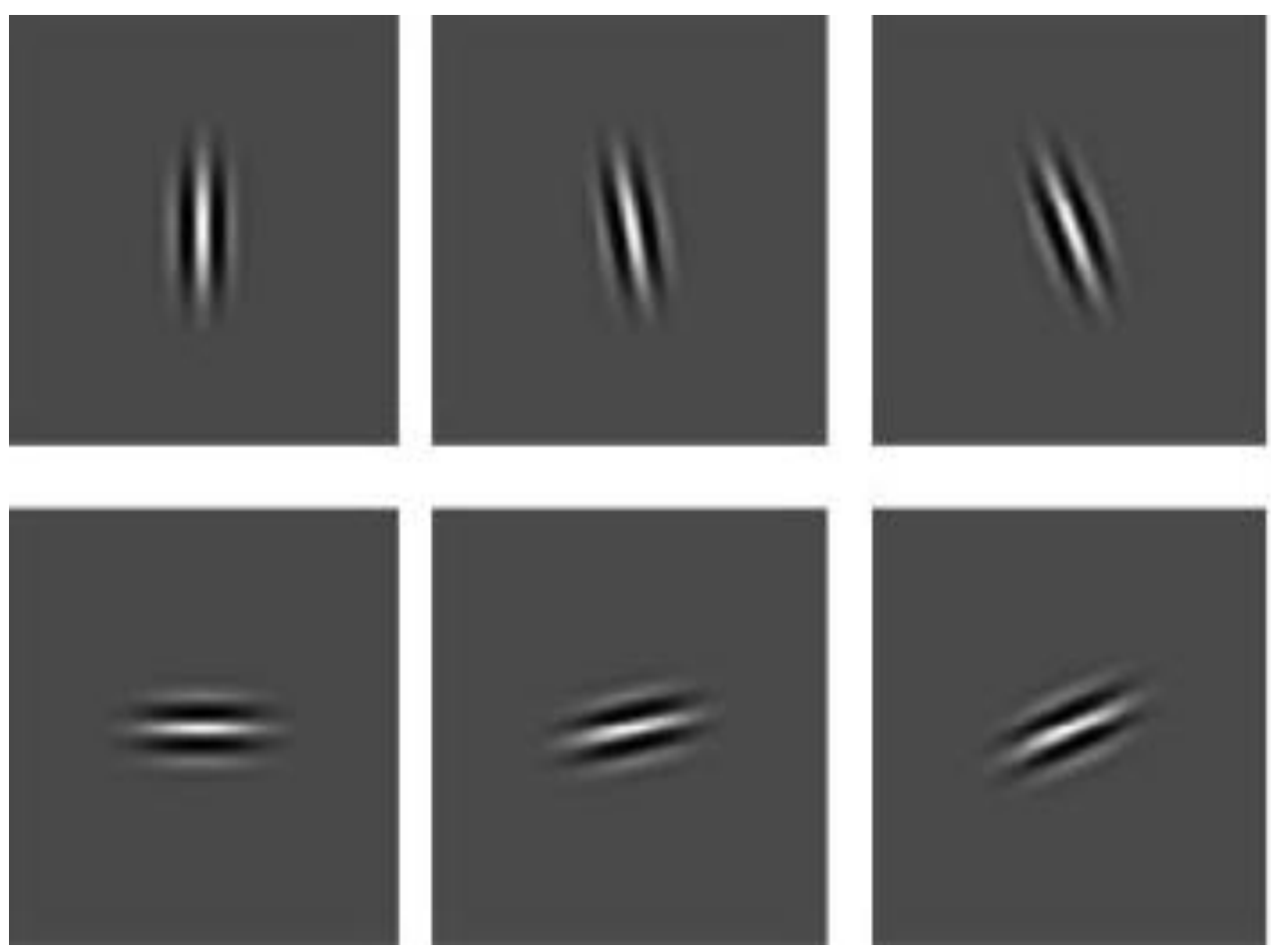

Fig. 5. 

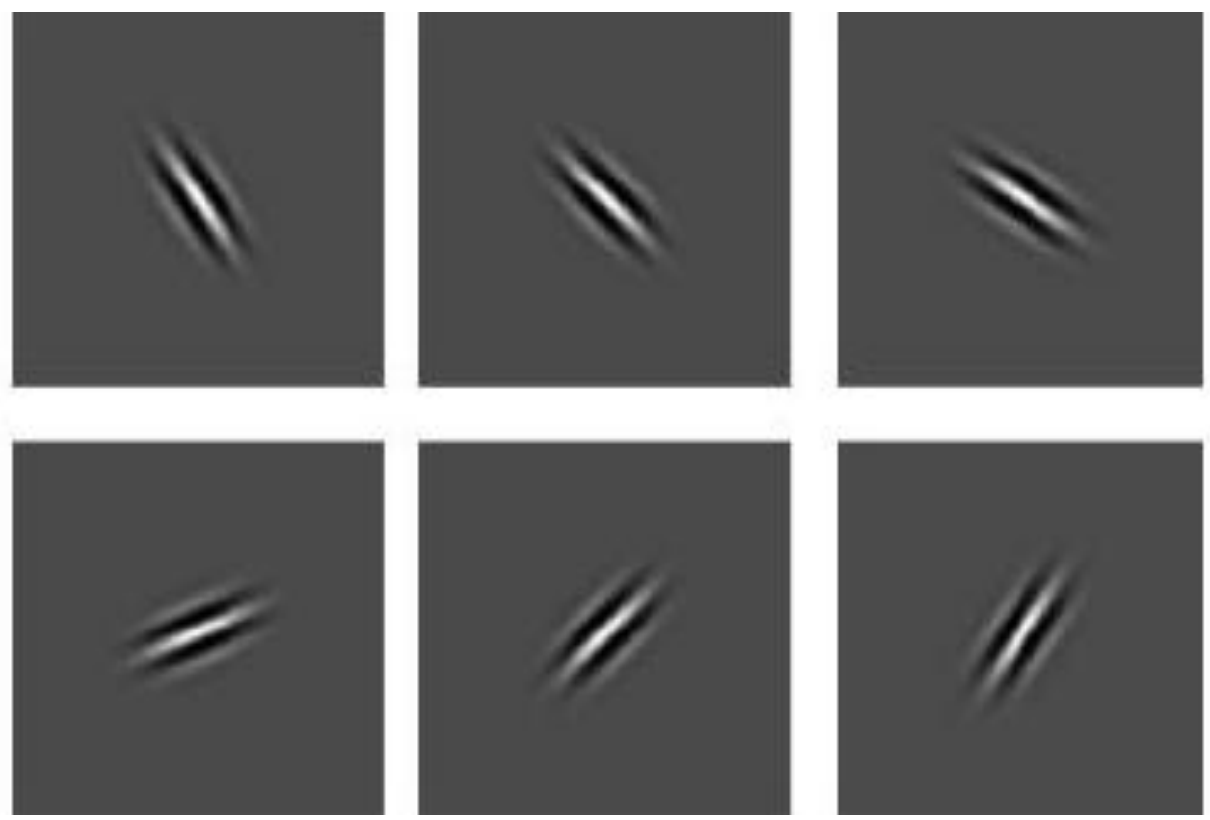

Fig. 6 .
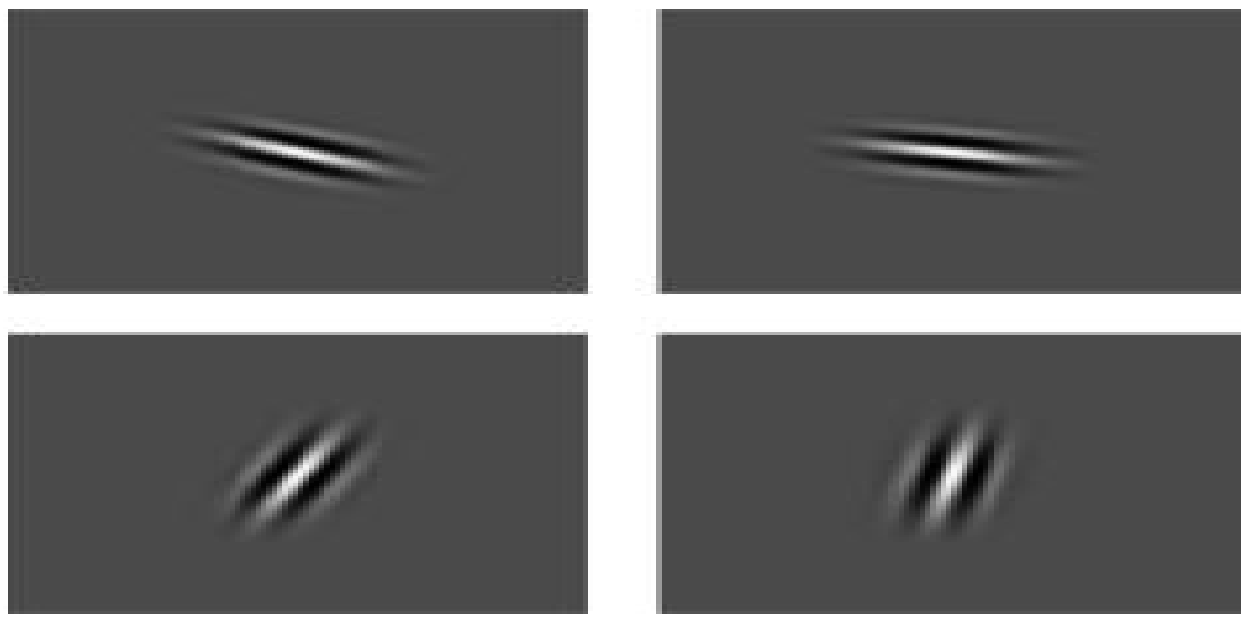

Fig. 7.

In image processing, the Gabor filter is typically used for edge selection, object contour detection, texture feature extraction, fingerprint image selection, local direction extraction, and other targets (Batra et al., 2004; Yang et al., 2003). 
In this paper, various orientations of the Gabor filter are used to improve the image of the fingerprint (Figs. 5 to 7). A sample result of applying the Gabor filter to the image of a fingerprint is shown in Fig. 8.

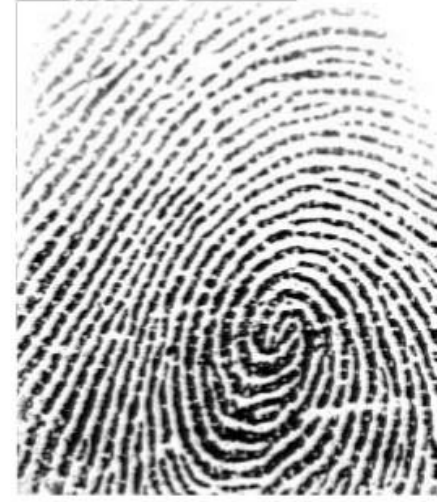

(a)

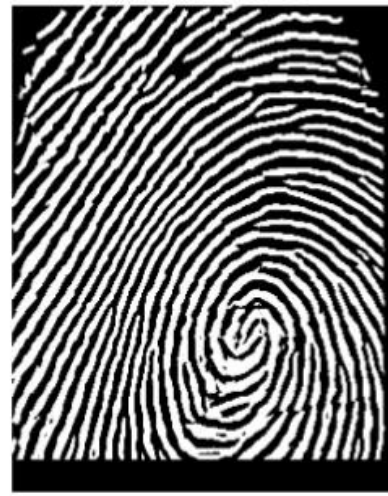

(b)

Fig. 8. Result of Gabor filters application:

a) original image of the fingerprint; b) image processed by the Gabor filter.

\subsection{Wavelet Transformation}

The main task in each type of image processing is to find an effective representation that enables a display in a compact form. In the modern theory and practice of signals, in particular in spectral analysis, signals of a special type of "wavelets" are used. In(Yang et al., 2003; Daubechies, 1990) decomposition of the image and extraction of its features for the classification of aircraft images based on the application of the Haar wavelet transformation and a multilayer neural network were presented. In this paper, the wavelet transformations of Haar and Daubechies are used to extract the features of the fingerprint image. A sample application of the $5^{\text {th }}$ level Daubechies wavelet transformation to extract the fingerprint image features is shown in Fig. 9.

The proposed method uses the Gabor filter to enhance the image, and the Haar and Daubechies wavelet transforms are used to extract the global features of the fingerprint image. To evaluate the efficiency of the feature extractions, wavelet transforms of the fifth and sixth levels are used. The neural network is created with 192 inputs for a wavelet transformation of the fifth level. The number of hidden neurons for this network varies from 200 to 250 . For a wavelet transformation of the sixth level, a neural network with 48 inputs is created, for which the number of hidden neurons varies from 80 to 120 . 
Both networks have seven outputs in accordance with the number of classification categories of fingerprints.

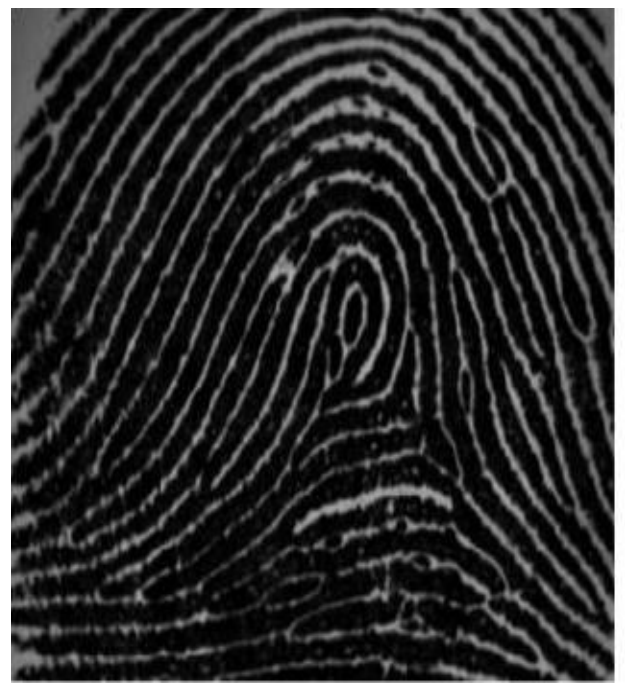

(a)

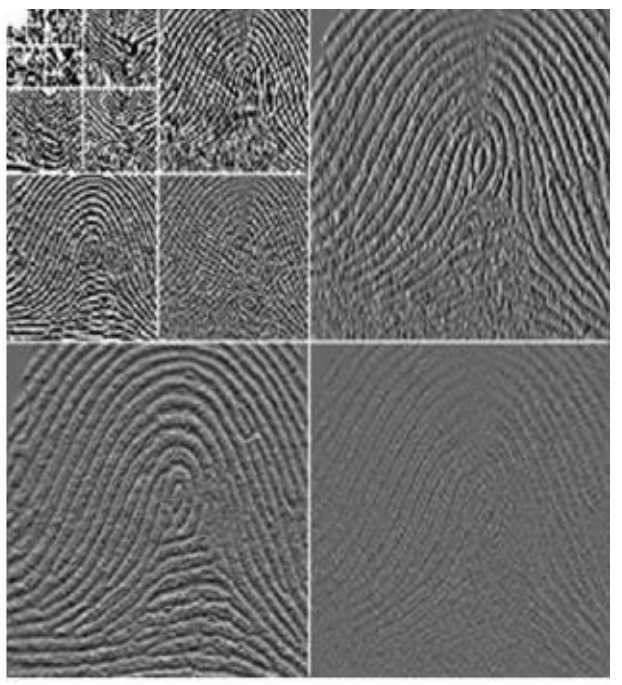

(b)

Fig. 9. Fingerprint feature extraction: a) original image; b) features extracted by Daubechies wavelet transformation.

The proposed method uses the Gabor filter to enhance the image, and the Haar and Daubechies wavelet transforms are used to extract the global features of the fingerprint image. To evaluate the efficiency of the feature extractions, wavelet transforms of the fifth and sixth levels are used. The neural network is created with 192 inputs for a wavelet transformation of the fifth level. The number of hidden neurons for this network varies from 200 to 250 . For a wavelet transformation of the sixth level, a neural network with 48 inputs is created, for which the number of hidden neurons varies from 80 to 120 . Both networks have seven outputs in accordance with the number of classification categories of fingerprints.

\section{Results and Discussion}

To test the algorithm performance, part of the FVC2006 fingerprint database(National Institute of Standards and Technology, 2017a; BCC Research, 2010; Jain and Uludag, 2003; Cole and Thumb, 2001; Jain et al., 1997) containing nine images of each category for learning (total $9 \times 7=63$ images) and 14 images of each category for testing (total 14 $\times 7=98$ images) were used. The comparison results of the extraction efficiency of the characteristics are shown in Figs. 10 and 11. 


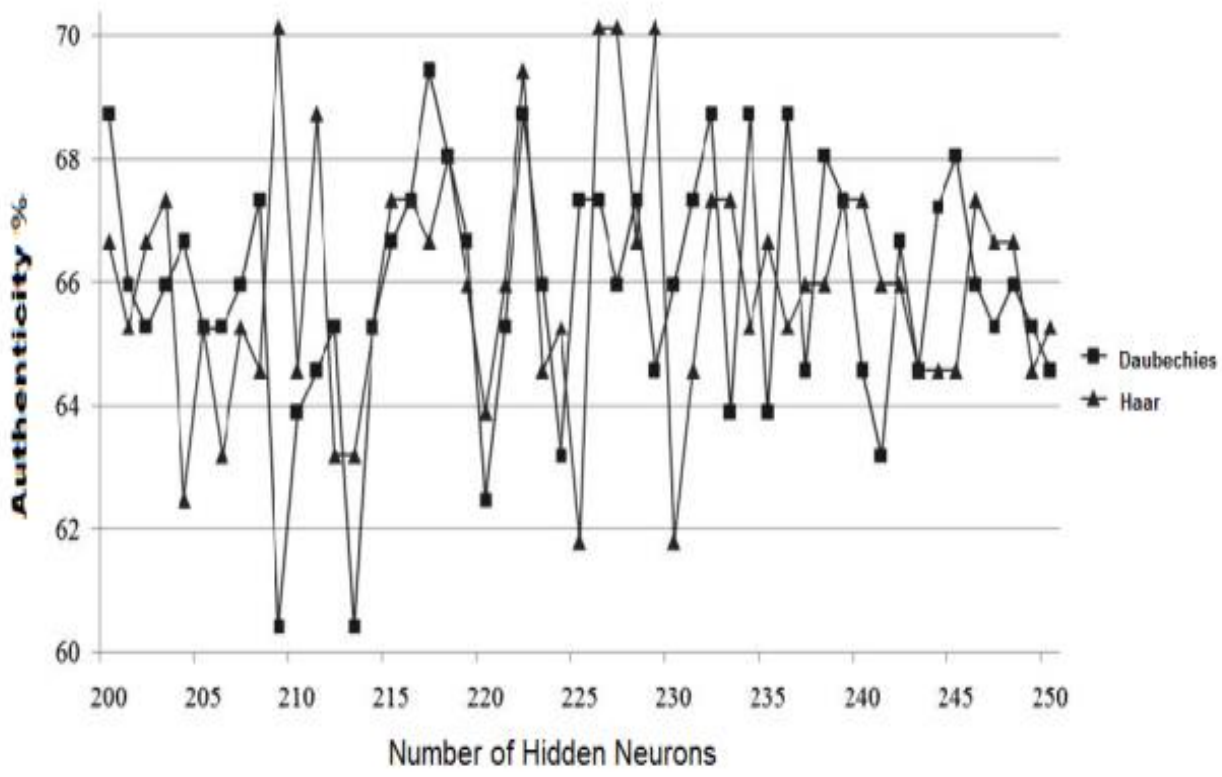

Fig. 10. Results comparison of Daubechies and Haar wavelet transforms level 5.

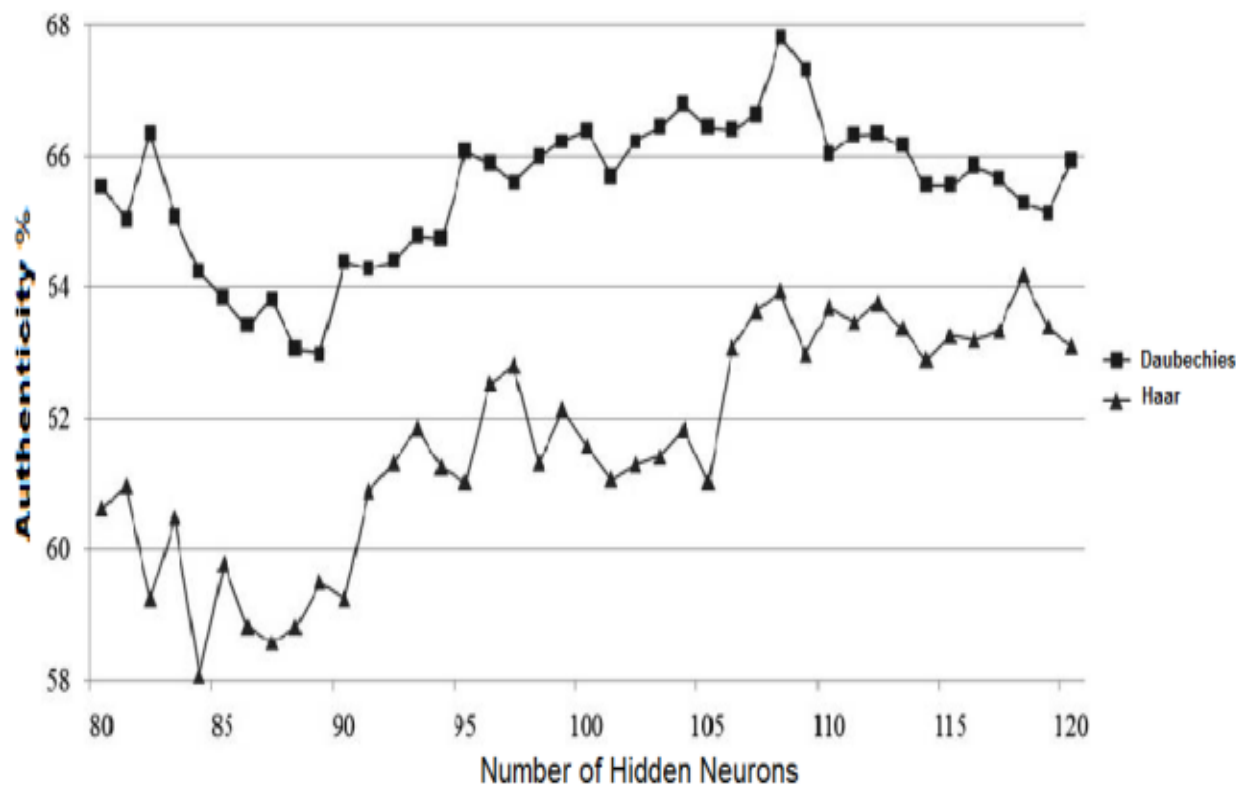

Fig. 11. Results comparison of Daubechies and Haar wavelet transforms level 6. 
An analysis of the results shows that the algorithm using the Daubechies wavelet transform yields better results than that based on the Haar wavelet. The algorithm efficiency using the Gabor filter is compared with the algorithm without using this filter in Figs. 12 and 13.

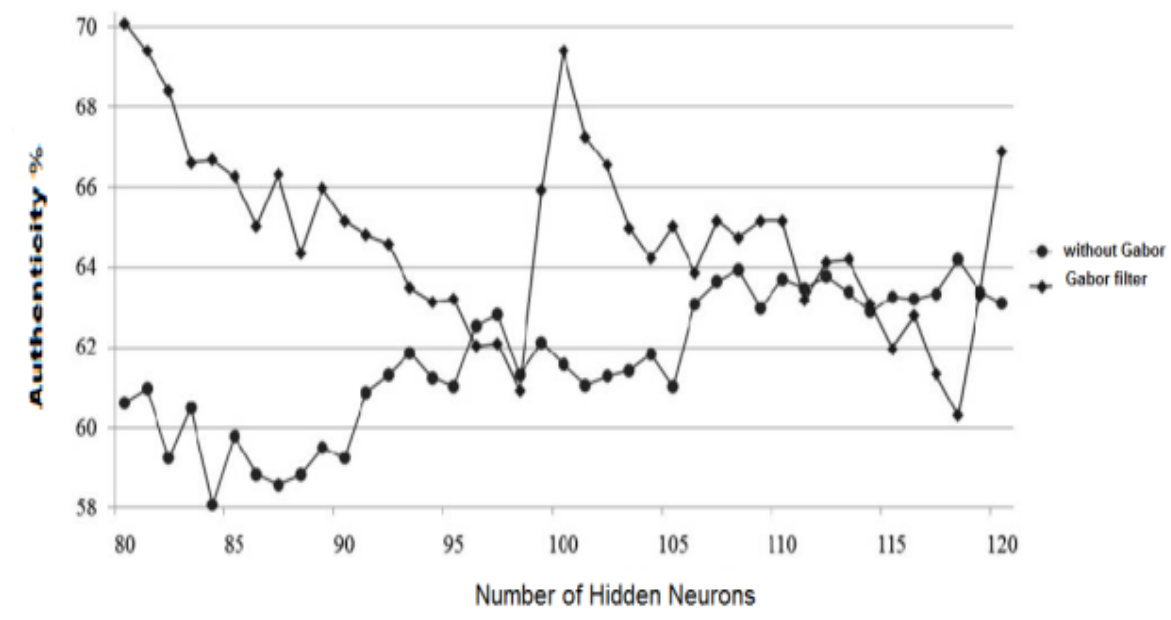

Fig. 12. Results comparison of the Haar wavelet transform, level 6, with and without the Gabor filter.

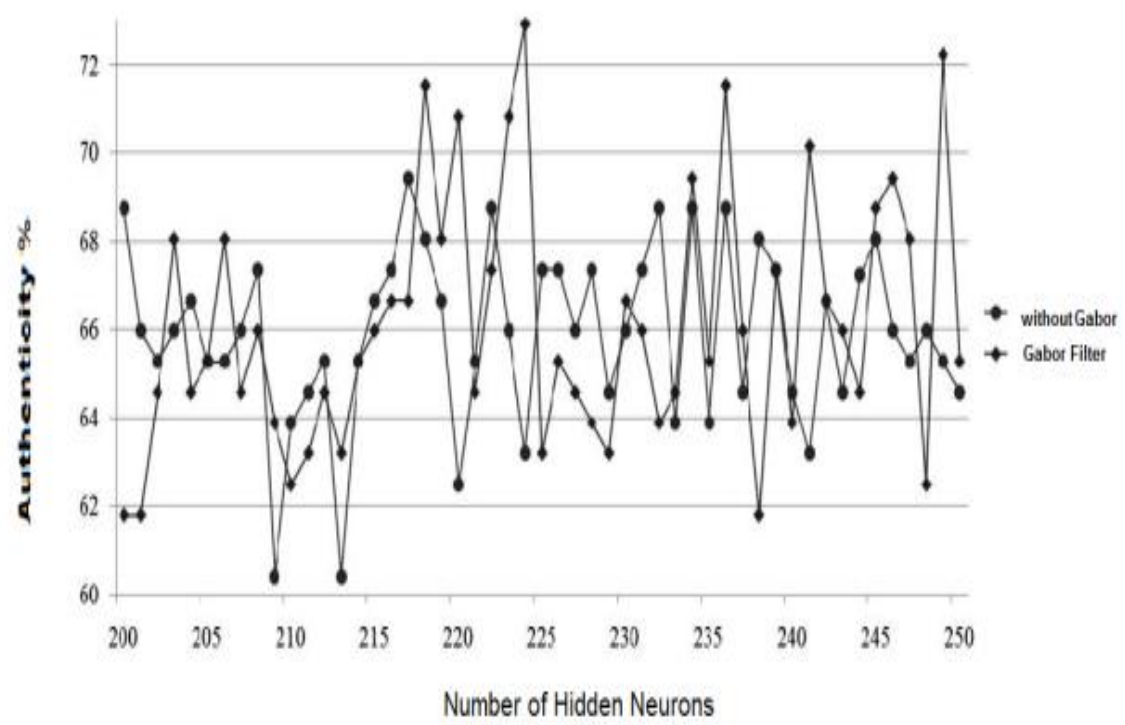

Fig. 13. Results comparison of the Daubechies wavelet transform, level 5, with and without the Gabor filter. 
The results were compared with the technique based on singularity on the same database by considering only 4 categories as shown in Table 1 . It can be seen that the main difference is the number of the images that cannot be recognized by the singularity technique is up to 254 and the whorl type is up to 125 . So the accuracy of the system with singularity based is only $87 \%$ for 4-category problem on the same data-base when images are of good quality, but when applied to images of poor quality the accuracy is less than $30 \%$. As shown in Table 2.

Table 1. The experimental results of singular based Technique for 4-category problem on quality images

\begin{tabular}{lccccc}
\hline \multirow{2}{*}{ True } & \multicolumn{5}{l}{ Exp. as } \\
\cline { 2 - 6 } & Whorl & $\begin{array}{c}\text { Left } \\
\text { loop }\end{array}$ & $\begin{array}{c}\text { Right } \\
\text { loop }\end{array}$ & Arch & Unknown \\
\hline Whorl & 602 & 20 & 23 & 10 & 125 \\
Left loop & 24 & 669 & 6 & 35 & 50 \\
Right loop & 23 & 10 & 689 & 46 & 36 \\
Arch & 18 & 33 & 19 & 1519 & 43
\end{tabular}

Table 2. The experimental results of singular based Technique for 4-class problem on poor Images

\begin{tabular}{|c|c|c|c|c|c|}
\hline \multirow[b]{2}{*}{ True } & \multicolumn{5}{|l|}{ Exp. as } \\
\hline & Whorl & $\begin{array}{l}\text { Left } \\
\text { loop }\end{array}$ & $\begin{array}{c}\text { Right } \\
\text { loop }\end{array}$ & Arch & Unknown \\
\hline Whorl & 25 & 2 & 2 & 0 & 71 \\
\hline Left loop & 5 & 15 & 1 & 1 & 58 \\
\hline Right loop & 1 & 7 & 10 & 10 & 81 \\
\hline Arch & 8 & 3 & 5 & 25 & 52 \\
\hline
\end{tabular}

The above results show that the algorithm using the Gabor filter works more efficiently than the algorithm without application of the filter when applied to images of low quality as shown in Table 3. 
Table 3. The experimental results of our method for 4-category problem on poor Images

\begin{tabular}{lrrrrr}
\hline & & & & & \\
\cline { 2 - 6 } True & Exp. as & & & & \\
\cline { 2 - 6 } & 40 & 7 & 5 & 3 & 45 \\
Whorl & 10 & 26 & 2 & 0 & 32 \\
Left loop & 10 & 10 & 37 & 12 & 40 \\
Right loop & 3 & 7 & 4 & 36 & 43 \\
Arch & & $\begin{array}{r}\text { Right } \\
\text { loop }\end{array}$ & Arch & Unknown
\end{tabular}

The average computing time of 1000 fingerprints is recorded at PC Core 2 Duo 2GHZ with 4 GB memory is less than one second and the system only spends about 0.3 second on the procedures of the classification as shown in Table 4.

Table 4. The computational Time of our system

\begin{tabular}{lc}
\hline Process & Time(s) \\
\hline Image enhancement & 0.58 \\
Feature extraction & 0.22 \\
Classification & 0.0002 \\
Total & 0.8002 \\
\hline
\end{tabular}

\section{Conclusion}

In this paper, an algorithm was proposed for the classification of latent fingerprint images by using papillary patterns based on the Gabor filter, wavelet transform, and multilayer neural network. The results showed that the combined application of the Gabor filter, 5th-level Daubechies wavelet transform, and multilayer neural network produced the best results.

\section{Acknowledgements}

The paper will be funded by Sarhad University of Science and Information Technology Peshawar, Pakistan 


\section{References}

Al-Nima, R. et al. (2016).Robust feature extraction and salvageschemes for finger texture based biometrics,IET Biometrics, 6(2),43-52.

Batra, D. et al.(2004). Gabor filter based fingerprint classification using support vector machines,IEEE India Ann. Conf.

BCC Research, Biometrics: Technologies and global Markets, Report. (2010), http://www.bccresearch.com/report/biometrics-technologies-markets-ift042c.html.

Cole, S., Thumb, R. (2001), SuspectIdentities: A History of Fingerprinting and Criminal

Identification,Harvard University Press,London.

Daubechies, I.(1990). Wavelet transform, time frequency localization and signal analysis,IEEE Trans. Inf. Theory,36, 961-1005.

Fingerprint Verification Competition 2002 Database (FVC2002), (2002).

Hsieh, C. T. (2009). An effective method for fingerprint classification,Tamkang J. Sci. Engin., 12(2), 169-182.

Jain, A. K. et al. (1997), An identity-authentication systemusing fingerprints,Proc. IEEE, 85(9), $1365-1388$.

Jain, A. K., Prabhakar, Hong, L.(1999). A multichannel approach to fingerprint classification.IEEE Trans. Pattern Anal. Mach. Intell.,21(4), 348-359.

Jain, A. K., Ross, A., Prabhakar, S. (2014).An Introduction to Biometric Recognition.IEEE Trans. Circuits Sys.Video. Technol.,14(1),4-20.

Jain, A.K.,Uludag, U. (2003), Hiding biometric data, IEEE Trans. Patt. Anal. Mach. Intell., 25(11),1494-1498.

Maltoni, D.,Maio, D., Jain,A. K., Prabhakar,S. (2009).Handbook of FingerprintRecognition,2nd Edition, Springer-Verlag.

Najim, E.,AlShemmary, A. (2012),Classification of fingerprint images using neural network technique, J. Eng., 1(3).

National Institute of Standards and Technology (NIST) (2017a) www.nist.gov/data/biomet.htm

National Institute of Standards and Technology (NIST) Special Database 27, (2017b).

Patil, S.R.,Suralkar,S.R. (2012).Fingerprintclassification using artificial neural network,Int. J. Emerg. Technol. Adv. Eng., 2(10).

Si, X. et al. (2015). Detection and rectification of distorted fingerprints,IEEE Trans. Pattern Anal. Mach. Intel.,37(3).

Wang, J. W. et al. (2015), Enhanced ridge structure for improving fingerprint image quality based on a wavelet domain, IEEE Sig. Process. Lett., 22(4).

Wilson, C. L., Candela,G. T., Watson, C. I. (1993). Neural network fingerprint classification, J. Artif. Neural Networks, 1, 203-228.

Yang, J.et al. (2003).A modified Gabor filter design method for figerprint image enhancement, Patt.Recog. Lett., 24, 1805-1817.

\section{Authors' information}

Hamid Jan, Engr., is the Headof the Department of Computer Science, Sarhad University of Science and IT Peshawar, Pakistan.

Amjad Ali, Dr., is Associate professor at the Department of Electrical Engineering, Sarhad University of Science and IT Peshawar, Pakistan.

Received September 12, 2017, revised February 12, 2018, accepted March 12, 2018 